SUBJECT AREAS: PROGNOSTIC MARKERS

SARCOMA

IMMUNOHISTOCHEMISTRY

DIAGNOSTIC MARKERS

Received

29 May 2013

Accepted

12 August 2013

Published

28 August 2013

Correspondence and requests for materials should be addressed to G.J.Z. Iguoi_zhang@ yahoo.com)

* These authors contributed equally to this work.

\section{Diagnostic and therapeutic strategy and the most efficient prognostic factors of breast malignant fibrous histiocytoma}

\author{
Si-Qi Qiu'* , Xiao-Long Wei ${ }^{1,2 *}$, Wen-He Huang ${ }^{1}$, Ming-Yao Wu², Yun-Sheng Qin ${ }^{3}$, Yang-Kang Li ${ }^{4}$ \\ \& Guo-Jun Zhang ${ }^{1,5}$
}

\begin{abstract}
'The Breast Center, Cancer Hospital of Shantou University Medical College, Guangdong, China, ${ }^{2}$ Department of Pathology, Shantou University Medical College, Guangdong, China, ${ }^{3}$ Department of Thoracic Surgery, Cancer Hospital of Shantou University Medical College, Guangdong, China, ${ }^{4}$ Department of Radiology, Cancer Hospital of Shantou University Medical College, Guangdong, China, ${ }^{5}$ Cancer Research Center, Shantou University Medical College, Guangdong, China.
\end{abstract}

We analyzed the clinicopathological features of 9 breast malignant fibrous histiocytoma (MFH) patients. Immunohistochemistry was used to make both diagnosis and differential diagnosis, and to identify prognostic factors. All tumors lacked epithelial markers but expressed mesenchymal markers, suggesting a mesenchymal origin. Of the five cases expressing Ki-67, two of three patients with axillary lymph node involvement died between 6-8 months, and two died at 17 and 26 months after diagnosis. The two remaining cases, with low Ki-67 expression, had no recurrent or metastatic disease at 145 months after diagnosis. Previous studies have shown that surgery is the primary treatment of choice, but no clear benefit from adjuvant chemotherapy was observed. We demonstrate that axillary lymph node involvement and high expression of Ki-67 are associated with poorer prognosis. A literature review indicates surgery remains the first choice for MFH, but benefits from adjuvant chemotherapy remain unclear.

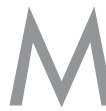
alignant fibrous histiocytoma (MFH) was first described as a distinct clinicopathologic entity by O'Brien and Stout in $1964^{1}$. The most frequent primary sites of MFH are the lower (49\%) and upper (19\%) limbs, and the retroperitoneum and abdomen (16\%). MFH of the breast is extremely rare, accounting for less than $1 \%$ of all breast primary malignancies ${ }^{2,3}$. So far, only 65 cases of breast MFH have previously been reported. The histogenesis of MFH still remains controversial ${ }^{4}$, making tumor morphology more important than histogenesis for diagnosis of MFH. Currently, MFH is considered to be an undifferentiated sarcoma. MFH tumors usually consist of fibroblast-like and histiocytic-like cells, mixed with pleomorphic giant and inflammatory cells ${ }^{2,5}$. According to the most recent WHO classification (2002 edition) of soft tissue tumors, MFH can be categorized into three morphological subtypes: storiform-pleomorphic MFH (undifferentiated high-grade pleomorphic sarcoma), giant cell MFH (undifferentiated pleomorphic sarcoma with giant cells), and inflammatory $\mathrm{MFH}$ (undifferentiated pleomorphic sarcoma with prominent inflammation).

A survey of the literature suggests that surgery is the first choice for treatment of breast MFH. However, the significance of chemotherapy and radiation therapy is not defined in the management of breast MFH due to the absence of prospective randomized trails ${ }^{6}$. A retrospective study of $200 \mathrm{MFH}$ patients by Weiss et al. reveals that deep, large tumors are associated with poor prognosis, while tumors with a prominent acute or chronic inflammatory component, or display highly myxoid forms of MFH have a better prognosis ${ }^{4}$. Blanchard et al. report that the median survival of patients with breast MFH is 58 months, but there is no correlation between the size of the primary tumor and the risk of recurrence or death from disease ${ }^{7}$. Due to the low incidence of the disease, the prognostic factors for breast MFH remain unclear.

For diagnosis, MFH needs to be distinguished from undifferentiated carcinomas and other sarcomas with a similar degree of cellular pleomorphism. Immunohistochemical staining is useful to exclude undifferentiated carcinomas as well as other sarcomas, and is widely used in clinical practice. Biological markers, especially Ki-67, are widely used as prognostic markers in breast cancers. Here, we review prior literature and analyze clinicopathological features and prognostic factors of 9 cases of breast MFH. Our immunohistochemistry results show that high expression of Ki-67 and axillary involvement are associated with poorer prognosis, and a literature review suggests that surgery remains the first choice for treatment of this disease. 


\begin{tabular}{|c|c|c|c|c|c|c|c|c|c|c|}
\hline $\begin{array}{l}\text { Case } \\
\text { No. }\end{array}$ & Age & $\begin{array}{l}\text { Site/Size } \\
(\mathrm{cm})\end{array}$ & Surgery & $\begin{array}{c}\text { Treatment } \\
\text { post-operation }\end{array}$ & $\begin{array}{l}\text { Lymph } \\
\text { nodes } \\
\text { involved }\end{array}$ & $\begin{array}{l}\text { Recurrence/ } \\
\text { Metastasis }\end{array}$ & $\begin{array}{l}\text { Treatment } \\
\text { after relapse }\end{array}$ & Re-recurrence & Treatment & Prognosis \\
\hline 1 & 68 & $\mathrm{~L} / 5$ & MRM & None & $0 / 12$ & No & & & & Alive \\
\hline 2 & 58 & $\mathrm{~L} / 15$ & MRM & None & $0 / 6$ & Lung & $\begin{array}{l}\mathrm{DDP}+\mathrm{THP}+ \\
\text { DTIC }\end{array}$ & & & $\begin{array}{c}\text { Deceased at } \\
6 \text { months }\end{array}$ \\
\hline 4 & 24 & $\mathrm{~L} / 5$ & MRM & $\mathrm{CAF}+\mathrm{CMF}$ & $0 / 4$ & No & & & & Alive \\
\hline 5 & 52 & $R / 13$ & MRM & CAF & $0 / 8$ & No & & & & Alive \\
\hline 6 & 20 & $\mathrm{~L} / 3$ & $\begin{array}{c}\text { LP followed by } \\
\text { MRM }\end{array}$ & None & $0 / 2$ & No & & & & Alive \\
\hline 7 & 73 & $\mathrm{~L} / 4$ & MRM & None & $3 / 8$ & $\begin{array}{l}\text { Chest ward } \\
\text { recurrence } \\
\text { (19 months } \\
\text { after } \\
\text { operation) }\end{array}$ & $\begin{array}{l}\text { LP + Skin-flap } \\
\text { reconstruction }\end{array}$ & $\begin{array}{l}\text { Three months } \\
\text { later, chest } \\
\text { ward } \\
\text { re-recurrence }\end{array}$ & $\begin{array}{l}\text { LP + Radiotherapy. } 1 \\
\text { One month later, } \\
\text { bilateral lung } \\
\text { metastasis with no } \\
\text { treatment. } \\
\text { Three months later, } \\
\text { died. }\end{array}$ & $\begin{array}{l}\text { Deceased at } \\
26 \text { months }\end{array}$ \\
\hline 8 & 51 & $\mathrm{~L} / 4$ & MRM & ADTIC & $0 / 13$ & $\begin{array}{l}\text { Chest ward } \\
\text { recurrence } \\
\text { (3 months } \\
\text { after } \\
\text { operation) }\end{array}$ & $\begin{array}{l}\mathrm{LP}+\text { Skin-flap } \\
\text { reconstruction }\end{array}$ & $\begin{array}{l}\text { Two months } \\
\text { later, chest } \\
\text { ward } \\
\text { re-recurrence } \\
\text { and bilateral } \\
\text { lung } \\
\text { metastasis }\end{array}$ & $\begin{array}{l}\text { IFO + DDP; } \\
\text { Bilateral lung } \\
\text { metastasis } \\
\text { progress, } \\
\text { Change to NP }\end{array}$ & $\begin{array}{c}\text { Deceased at } \\
8 \text { months }\end{array}$ \\
\hline 9 & 48 & L/17 & $\begin{array}{l}\text { RM + Skin-flap } \\
\text { reconstruction }\end{array}$ & $\mathrm{IFO}+\mathrm{EPI}$ & $0 / 10$ & No & & & & $\begin{array}{l}\text { Deceased at } \\
17 \text { months }\end{array}$ \\
\hline \multicolumn{11}{|c|}{$\begin{array}{l}\text { MRM: Modified radical mastectomy; RM: Radical mastectomy; LP: Lumpectomy. } \\
\text { CAF: Cyclophosphamide + Adriamycin + Fluorouracil. } \\
\text { CMF: Cyclophosphamide + Methotrexate + Fluorouracil. } \\
\text { ADTIC: Adriamycin + Dacarbazine. } \\
\text { NP: Navelbine + Cisplatin. } \\
\text { IFO: Ifosfamide; EPI: Epirubicin; DDP: Cisplatin. } \\
\text { DTIC: Dacarbazine; THP: Pirarubinci. }\end{array}$} \\
\hline
\end{tabular}

\section{Results}

Clinical characteristics of patients. The detailed information on each patient is listed in Table 1 . All 9 patients presented with a painless breast mass. The tumor mass was located in the left breast in 8 cases. A typical clinical finding is shown in Figure 1, and imaging of the breasts in the same patient is shown in Figure 2. Of the 9 cases, mean tumor size was $7.9 \mathrm{~cm}$ in diameter, but the median tumor diameter was $5 \mathrm{~cm}$. All patients received surgery, and 7 cases underwent modified radical mastectomy. One underwent lumpectomy followed by modified radical mastectomy, and one was treated with radical mastectomy + skin-flap reconstruction. Axillary lymph node dissection revealed lymph node involvement in 2
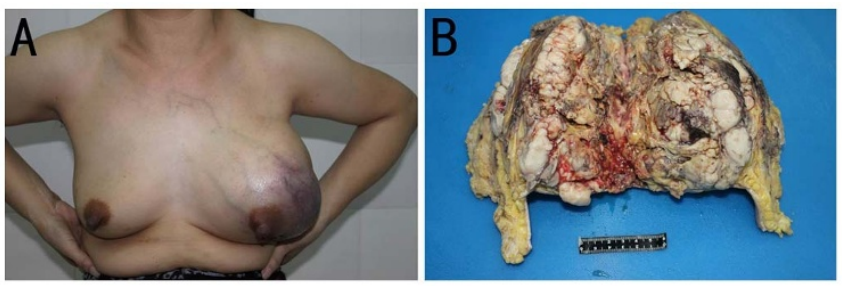

Figure 1 Clinical and pathological findings in a patient with breast MFH. (A) The breasts were asymmetrical with the left breast enlarged. A protuberance and irregular mass were observed in the outer half of the left breast, with dark blue skin on the surface of the breast. Subcutaneous blood vessels are tremendously dilated. (B) The gross tumor occupied most of the breast parenchyma in formalin-fixed samples. The cut surface of the tumor was grayish and fleshy, with areas of cystic change, necrosis and hemorrhage. The patient shown in Fig. 1 has permitted us to publish her information in Scientific Reports. cases, whereas lymph nodes in the remaining 7 cases appeared metastasis-free. Five cases had adjuvant chemotherapy, and 4 cases did not receive further treatment. One patient who had previous contralateral chest wall radiation therapy developed contralateral breast MFH 4 years after the modified radical mastectomy.

During the follow-up, 2 cases had local recurrence and another 2 cases were found to have distant metastases with one in the lung and the other in the liver. The patient with lung metastasis died 6 months after operation, while the patient with liver metastasis died 7 months after operation. Both patients with local recurrence were further surgically-treated with local excision. The two cases, that experienced chest wall re-recurrence and lung metastases at 2 and 3 months after the local excision, received supportive treatment and/or palliative therapy, and died of lung metastases at 8 and 26 months after primary surgery. One case died of tumor burden 17 months after diagnosis.

Pathological findings. H/E-stained slides were independently reviewed by two pathologists (M.W., X.W.). Diagnosis was based on the WHO criteria (2002 edition). Seven cases were classified as storiform-pleomorphic MFH (Figures 3A and B), as they showed a classical microscopic appearance of apparent pleomorphic tumor cells and nuclei, mixed heteromorphic giant cells, spindle cells and round histiocytic-like cells. One case was diagnosed as inflammatory MFH (Figure 3C), as the tumor consisted of abundant benign and malignant xanthoma cells, atypical spindle cells, and acute and chronic inflammatory cells. One case was classified as the giant cell type (Figure 3D), as the tumor showed an osteoclast-like giant cell reaction except for the pleomorphic elliptical and spindle cells. Lipoblasts were not seen in any of the 7 cases examined. Neither epithelial components nor normal ductal components were observed in 

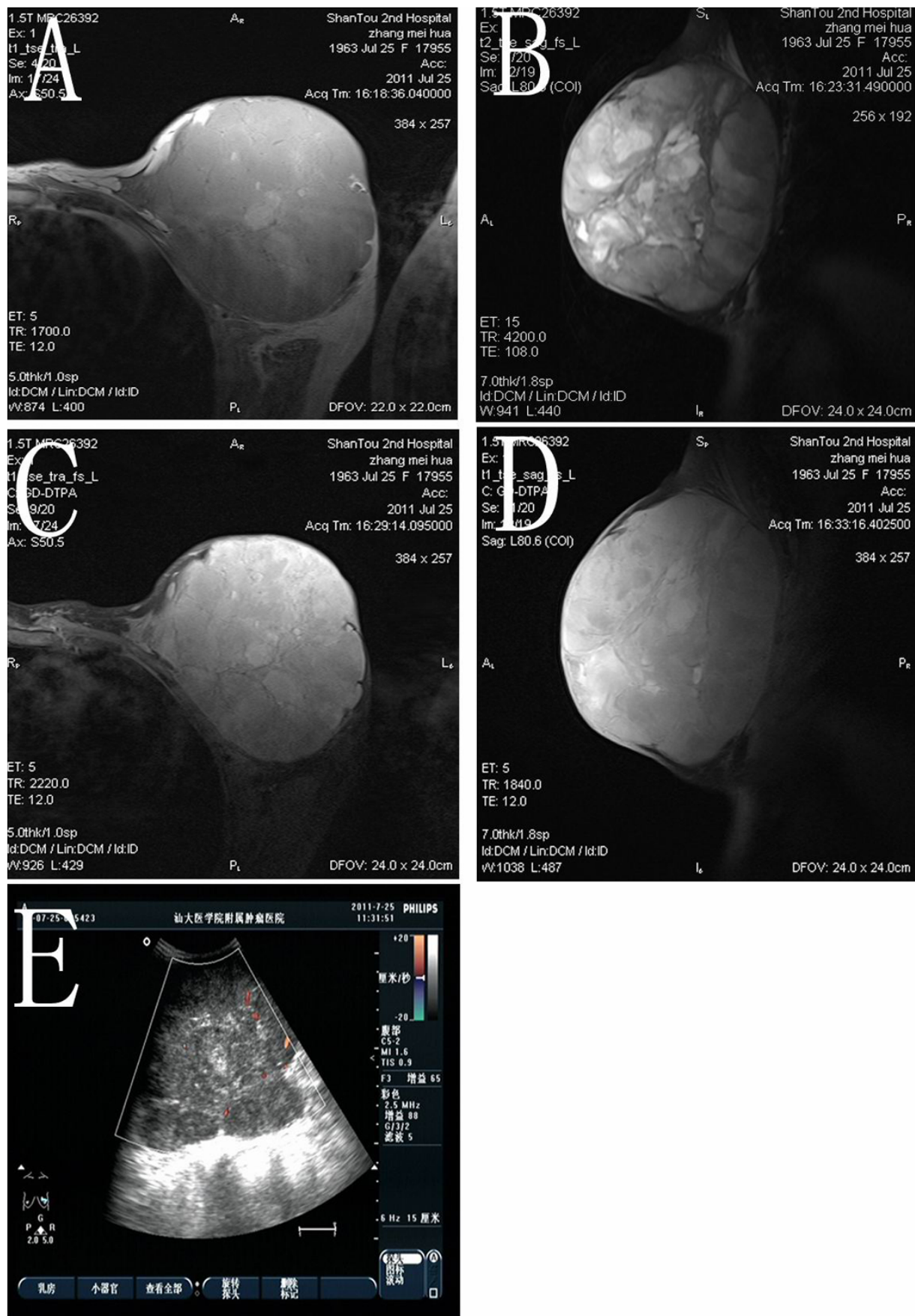

Figure $2 \mid$ Imaging results of the same patient as in Figure 1. (A, B) MRI findings: A giant mass, $10.5 \times 13.0 \times 13.5 \mathrm{~cm}$ in size, was detected in the left breast. The majority of the lesion showed relatively clear edges and a lower signal than normal breast tissue on transverse T1- and sagittal T2-weighted images. Multiple focal hemorrhages manifested as high signal intensities on both T1- and T2-weighted images. (C, D) Obvious asymmetric enhancement was noted on transverse and sagittal T1-weighted images obtained using a fat tissue suppression technique following intravenous contrast administration. (E) Ultrasound imaging of the left breast demonstrates a mass of low echogenicity with a non-homogeneous internal echo pattern. Color Doppler sonogram reveals hypervascularity in the breast mass.

any of the tumors of the 9 cases. No special proliferating pattern was apparent.

IHC staining. Immunohistochemistry was performed on tissues from 7 patients. Immunoreactivities for ER, PR, Her-2, desmin, cytokeratin (AE1/AE3 and CK5/6), and EMA were negative, while those for vimentin, CD163 and AACT were positive (Figure 4). P53 expression was positive in 2 cases, and VEGF expression positive in 4 cases. Ki-67 and CD68 were each detected in 5 cases (Figure 4). SMA was focally positive in 4 cases (Figure 4). We also performed S-100 immunostaining to investigate if the tumors originated from neural tissues. Interestingly, S-100 was focally expressed in one patient (Figure 4), similar to a prior report by Choudhury.

Tumors from the 5 patients who had a short life span, including the 2 that originally presented with axillary lymph node involvement, all highly expressed Ki-67. Currently, both of the remaining 2 cases, originally having low Ki-67 expression, are still alive without evidence of local recurrence or distant metastasis at follow up of 145 months after diagnosis. The IHC staining results are listed in Table 3. 

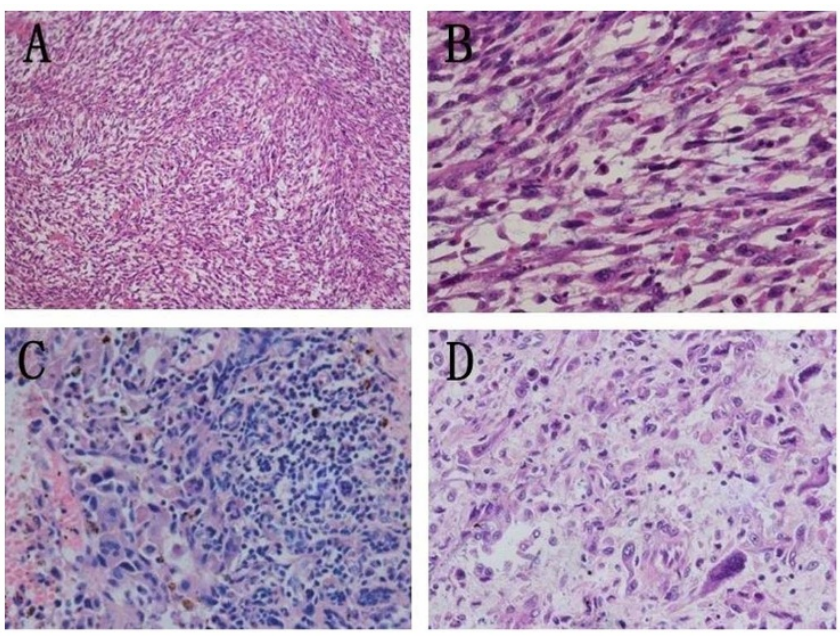

Figure 3 Pathological findings of 3 MFH subtypes. Storiformpleomorphic subtype: (A) The tumor was composed of hypercellular spindle cells arranged in a characteristic storiform pattern (H\&E 100X). (B) Amplification of Figure 3A, shows atypical, highly proliferating cells (H\&E $400 \times)$. Inflammatory subtype: (C) Proliferation of fibroblasts and multinucleated histiocytes. Numbers of inflammatory cells were seen in the interstitial substance (H\&E $400 \times)$. Giant cell subtype: (D) Numbers of spindle-shaped or tadpole-shaped, multinucleated giant cells were seen. Cell nuclei ranged from 2 to 14 , and the shape was irregular (H\&E 400×).

\section{Discussion}

Breast MFH has been reported to account for less than $1 \%$ of all primary malignancies of the breast ${ }^{2,3}$. In our series, a total of 9 cases of breast MFH are reported, equal to a frequency of $0.2 \%$ (9/3981) of breast malignancies treated in our hospital. Of the 9 cases, 8 cases were diagnosed as primary MFH of the breast.
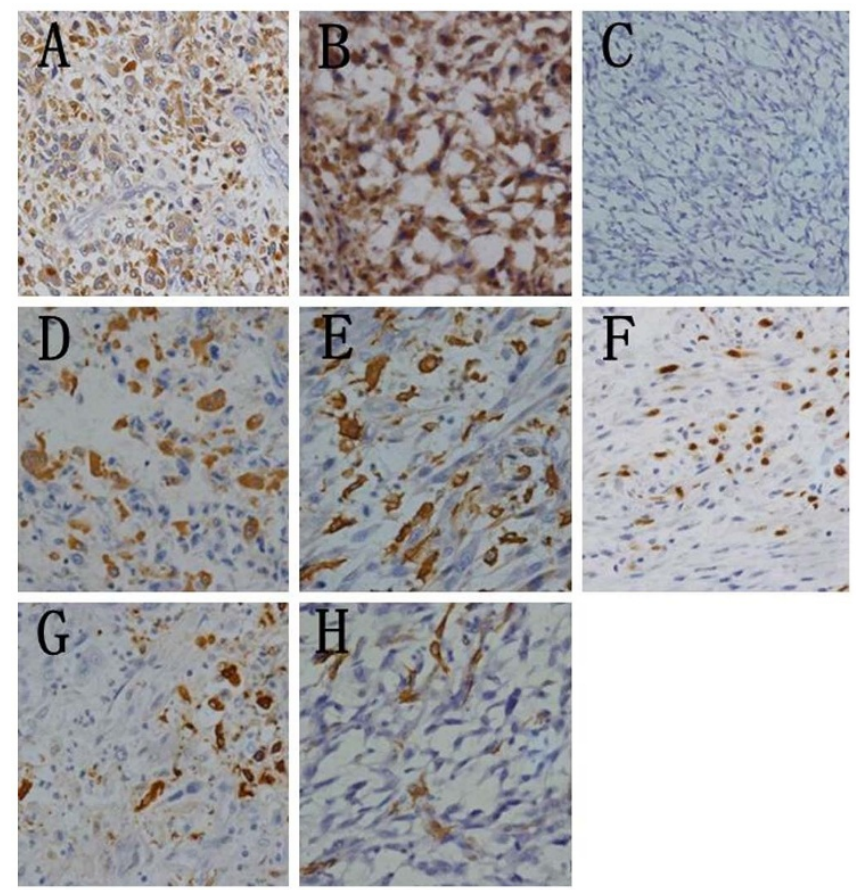

Figure 4 Immunohistochemical staining. Representative images show positive expression for AACT (A) vimentin (B) CD68 (D) CD163 (E) Ki67 (F) S-100 (G) and SMA (H) and negative expression for cytokeratin AE1/AE3 (C). (Immunohistochemistry $400 \times$ ). Ki-67 staining was considered positive if immunostaining was seen in more $(>)$ than $10 \%$ of the tumor nuclei.
Differential diagnosis for MFH is a challenge because its morphological features are complex. In this study, H\&E staining showed that all cases consist of fibroblast-like and histiocytic-like cells, supporting an undifferentiated tumor. Extensive sampling found neither invasive ductal carcinoma nor normal ductal components. Lipoblasts, whose presence is necessary for the diagnosis of liposarcoma, are not seen in any of the 7 cases, enabling the exclusion of liposarcoma. Leiomyosarcoma usually has small areas with conventional smooth muscle cytomorphology and a fascicular growth pattern. Additionally, leiomyosarcoma usually shows positive expression of desmin and SMA in the fascicular spindle cell component. In our study, although 4 cases focally expressed SMA, all were negative for expression of desmin. Moreover, there was no conventional smooth muscle cytomorphology and a fascicular growth pattern observed. Thus, leiomyosarcoma was ruled out. Rhabdomyosarcoma can also be excluded, as neither alveolar structure nor striated muscle structure was found in the tumor. Besides, all the tumors were negative for desmin, a specific marker for rhabdomyosarcoma. Pure osteosarcoma is extremely rare and many cases of this disease show osteosarcomatous elements within metaplastic carcinomas. In this regard, osteosarcoma was not considered. In one case, the neural marker S100 was focally positive, showing an ability to differentiate into neurogenic tissue. Nevertheless, neurogenic sarcoma was ruled out because most of the tumor cells were still in an undifferentiated state. Therefore, MFH can be differentiated from other possible tumors with similar pathological features, including malignant phyllodes tumor (MPT), metaplastic carcinoma, pleomorphic leiomyosarcoma, osteosarcoma and pleomorphic rhabdomyosarcoma, based on histopathological diagnosis and immunohistochemical staining.

It has been demonstrated that MFH of the breast progresses quickly with a high recurrence rate. IHC staining shows that tumors, in all 7 cases examined, fail to express epithelial markers like EMA and cytokeratin (AE1/AE3 and CK5/6), suggesting a non-epithelial genesis, whereas strong positive staining for vimentin, a specific marker for mesenchymal cellular differentiation, strongly supports the mesenchymal origin of these tumors. Post-radiation sarcoma includes osteosarcoma, extra-breast soft tissue $\mathrm{MFH}^{9}$, and sarcomas within the irradiated fields ${ }^{10}$. In addition, the criteria for radiationinduced sarcoma includes a prior history of radiation, latency of 3-4 years or more, development of the sarcoma near to or within a previously irradiated field, and a histological diagnosis of sarcoma $^{11,12}$. Here, we report one case of contralateral breast MFH followed by adjuvant radiotherapy for breast cancer. We consider it a radiation-induced MFH because the diagnosis meets the diagnostic criteria mentioned above, and it is similar to a previously reported case $^{12}$.

A literature search reveals that surgical treatment is still the treatment of choice for breast $\mathrm{MFH}^{6}$. There are several surgical options for breast $\mathrm{MFH}$, including radical mastectomy, mastectomy, and breast conserved surgery ${ }^{6,13}$. The essentiality of axillary lymph node dissection is still unknown. Some reports strongly recommend the procedure ${ }^{2,14}$, while others do not ${ }^{3,6,13}$. In our cohort, 8 patients out of 9 underwent modified radical mastectomy. In the remaining patient, skin flap reconstruction was immediately performed after radical mastectomy because of the large size of the tumor mass, being 17 $\mathrm{cm}$ in diameter with invasion into the pectoralis major and skin covering the breast. Two patients had axillary lymph node involvement, giving an axillary lymph node positive rate of $22.2 \%$, which is similar to previous reports ${ }^{2,14}$. The two patients, who initially presented with positive nodes, displayed worse prognosis. Therefore, axillary lymph node dissection appears to be very important for patients with axillary involvement, and for the prediction of prognosis. MFH cells rarely spread via lymphatic vessels, and almost $80 \%$ of breast $\mathrm{MFH}$ patients show negative lymph nodes. Thus, axillary lymph node dissection may be over-treatment for most breast MFH patients. As validated in early breast cancer patients, 
Table 2 | Primary antibodies used for immunohistochemistry

\begin{tabular}{llcc} 
Antigen & \multicolumn{1}{c}{ Clone No. } & Antibody Source & Dilution \\
\hline AACT & Polyclonal & Abcam (Cambridge/UK) & $1: 100$ \\
EMA & E29 & Lab Vision (Kalamazoo/USA) & $1: 200$ \\
Vimentin & V9 & Invitrogen (Carlsbad/USA) & $1: 100$ \\
CK & AE1/AE3, CK5/6 & Invitrogen (Carlsbad/USA) & $1: 100$ \\
Ki-67 & K-2 & GBI (Mukilteo/USA) & $1: 100$ \\
S-100 & $4 c 4.9$ & Lab Vision (Kalamazoo/USA) & $1: 50$ \\
ER & EP1 & Epitomics (Burlingame/USA) & $1: 100$ \\
PR & EP2 & Epitomics (Burlingame/USA) & $1: 200$ \\
Her-2 & EP1045Y & Lab Vision (Kalamazoo/USA) & $1: 100$ \\
VEGF & VG1 & Lab Vision (Kalamazoo/USA) & $1: 200$ \\
Desmin & ZC18 & Invitrogen (Carlsbad/USA) & $1: 50$ \\
SMA & IA4 & Abcam (Cambridge/UK) & $1: 50$ \\
CD163 & 10D6 & Neomarkers (Kalamazoo/USA) & $1: 50$ \\
CD68 & PG-M1 & Neomarkers (Kalamazoo/USA) & $1: 100$ \\
P53 & DO-7 & Dako (Glostrup/Denmark) & $1: 200$ \\
\hline
\end{tabular}

sentinel lymph node biopsy may be an appropriate option to determine the course of treatment for breast MFH, with axillary lymph node dissection performed only in patients with positive sentinel lymph nodes.

The role of adjuvant therapy for MFH is uncertain due to the lack of prospective randomized clinical trails ${ }^{6,15}$. Of the 5 cases that received adjuvant chemotherapy in the study, 2 patients died of either liver or lung metastasis at 7 and 8 months after diagnosis (cases No. 3 and 8, Table 1), one patient died of tumor burden 17 months after diagnosis, and the remaining 2 patients are still alive without evidence of recurrence/metastasis (Case No. 4 and 5, Table 1). While among 4 patients without any post-operative adjuvant therapy, one died of lung metastasis 6 months after diagnosis (Case No. 2, Table 1), one died of lung metastasis eventually after repeated chest wall recurrence (Case No. 7, Table 1), and the other two are still alive without any evidence of recurrence/metastasis (Case No. 1 and 6, Table 1). The lack of clinical benefit from adjuvant chemotherapy could possibly be due to the relatively large tumor size in the chemotherapy group. MFH patients do not respond to endocrine therapy due to the lack of ER/PR expression ${ }^{6}$, and the effectiveness of radiation therapy is not clear ${ }^{15}$. Van Niekerk et al. find that preoperative radiotherapy could achieve slight regression of both the primary tumor and metastasis to the axillary lymph node ${ }^{14}$. However, Blanchard does not recommend radiotherapy after a clear cancerfree surgical margin operation ${ }^{7}$. In the present study, no patient underwent adjuvant radiotherapy, although local recurrent disease was treated with radiation in one patient who later developed bilateral lung metastasis.

Prognostic factors for MFH of the breast remain unclear because of the small number of cases presented and the short follow-up period in most studies ${ }^{16}$. To investigate the prognostic significance of tumor biomarkers, immunohistochemical detection of Ki-67, VEGF, ER, PR, Her-2 and p53 was performed on 7 available cases. Four out of 5 patients with high Ki-67 expression, two of whom suffered from local recurrence, developed distal metastasis, and had a life span of 6 to 26 months. In 2 patients with low Ki-67 expression, no recurrence/distal metastasis was found in the follow-up at 145 months. This indicates that patients with high Ki-67 expression have a dramatically shorter life span than those with low Ki-67 expression, and suggests that elevated expression of Ki-67 is associated with local recurrence, distant metastasis, and poor prognosis in MFH of the breast. The present study does not show prognostic significance of either p53 or VEGF expression. Taken together, our results suggest that a high proliferation index, rather than angiogenesis or apoptosis, contributes to the rapid progression and poorer prognosis in breast MFH patients. In addition to surgery, we recommend that the patients with high Ki-67 expression should be adjuvantly treated with systemic therapy.

In summary, we report 9 cases of breast MFH, analyze the clinicopathological characteristics of the patients, and provide a literature review. Surgical treatment remains the first choice for MFH of the breast, and axillary sentinel lymph node biopsy may be used to decide whether axillary lymph node dissection should be performed. Patients with axillary lymph node involvement and high expression of Ki-67 can be expected to display early local recurrence, distant metastasis, and poorer prognosis.

\section{Methods}

Patients. Between 1994 and 2011, 9 patients with breast MFH were admitted to the Affiliated Cancer Hospital of Shantou University Medical College. All patients were female, with ages ranging from 20 to 73 years old and a median age of 58 years. The clinicopathological features of the patients are summarized in Table 1. All patients were followed-up either by telephone, or by regular clinic visitation, every 3-6 months for the first 2 years, every 6 months for the 3rd to 5 th year, and every year thereafter following primary surgical treatment.

Hematoxylin \& Eosin staining. Complete sectioning was performed for all the patients to ensure a precise diagnosis. $4 \mu \mathrm{m}$-thick formalin-fixed and paraffin embedded sections were prepared for H\&E staining. Briefly, paraffin embedded sections were deparaffinized and rehydrated in a series of xylene and ethanol baths of decreasing concentration. Slides were put in hematoxylin solution for $1 \mathrm{~min}$, followed by $1 \%$ alcoholic hydrochloric acid for 3 seconds, and then eosin solution for $1 \mathrm{~min}$.

Immunohistochemistry (IHC). $4 \mu \mathrm{m}$-thick formalin-fixed and paraffin embedded sections were prepared for immunohistochemistry. Vimentin, desmin, smooth muscle actin (SMA), cytokeratin (AE1/AE3 and CK5/6), epithelial membrane antigen (EMA), alpha-1-antichymotrypsin (AACT), estrogen receptor (ER), progesterone receptor (PR), human epidermal growth factor receptor 2 (Her-2), vascular endothelial growth factor (VEGF), p53, S-100, CD163, CD68 and Ki-67 were detected in the present study. Immunohistochemistry was performed as previously described ${ }^{17}$. Briefly, paraffin embedded sections were deparaffinized and rehydrated in a series of xylene and ethanol baths of decreasing concentration. Endogenous peroxidase activity was blocked with $0.5 \% \mathrm{H}_{2} \mathrm{O}_{2}$ for $30 \mathrm{~min}$, followed by antigen

Table 3 | Immunohistochemical results in 7 cases of breast MFH

\begin{tabular}{|c|c|c|c|c|c|c|c|c|c|c|c|c|c|c|c|c|c|}
\hline & ER & PR & Her-2 & $\mathrm{Ki}-67$ & VEGF & P53 & Vim & AACT & Des & SMA & S-100 & CK & CK5/6 & EMA & CD68 & CD163 & Survival \\
\hline 2 & - & - & - & $12 \%$ & + & - & + & + & - & $\mathrm{F}+$ & - & - & - & - & - & + & $\begin{array}{l}\text { Deceased } \\
\text { at } 6 \text { months }\end{array}$ \\
\hline 3 & - & - & - & $>50 \%$ & - & - & + & + & - & $\mathrm{F}+$ & - & - & - & - & - & + & $\begin{array}{l}\text { Deceased } \\
\text { at } 7 \text { months }\end{array}$ \\
\hline 4 & - & - & - & - & - & - & + & + & - & - & - & - & - & - & + & + & Alive \\
\hline 7 & - & - & - & $25 \%$ & - & - & + & + & - & $\mathrm{F}+$ & - & - & - & - & + & + & $\begin{array}{l}\text { Deceased } \\
\text { at } 26 \text { months }\end{array}$ \\
\hline 9 & - & - & - & $40 \%$ & ++ & - & + & + & - & $\mathrm{F}+$ & - & - & - & - & + & + & $\begin{array}{l}\text { Deceased } \\
\text { at } 17 \text { months }\end{array}$ \\
\hline
\end{tabular}


retrieval by boiling for $20 \mathrm{~min}$ in sodium citrate buffer, $\mathrm{Ph}$ 6.0. Nonspecific binding was blocked by incubating the tissue sections with $4 \%$ BSA in PBS for 30 min. Slides were incubated overnight at $4{ }^{\circ} \mathrm{C}$ with primary antibody, followed by incubation with secondary antibody for $60 \mathrm{~min}$ at room temperature. All slides were developed with diaminobenzidine followed by hematoxylin counterstaining. Omission of the primary antibody was used as a negative control and resulted in lack of specific staining. All antibodies used in the study are summarized in Table 2.

Immunostaining was evaluated independently by two pathologists (X.W., M.W.). $\mathrm{ER}^{18}, \mathrm{PR}^{18}, \mathrm{p}^{19} 3^{19}$ and $\mathrm{Ki}-67$ staining was considered positive if immunostaining was seen in more than $10 \%$ of tumor nuclei. Positive staining $(3+)$ for Her-2 corresponded to strong staining in $>30 \%$ of tumor cells, whereas weak to moderate staining in $>10 \%$ cells was scored as equivocal $(2+)$, and no staining or faint incomplete staining was scored as negative $(-)$ or faint $(1+)^{20}$. For VEGF staining, intensity of cytoplasmic immunostaining was scored from 0 to $3+$ (no staining to strong staining, respectively) ${ }^{21}$. Vimentin, AACT, desmin, S-100, CK, EMA, CD68 or CD163 staining was considered positive if cell staining was either in the cytoplasm, membrane, or nucleus.

1. O’Brien, J. E. \& Stout, A. P. Malignant Fibrous Xanthomas. Cancer. 17, 1445-55 (1964).

2. Iellin, A., Waizbard, E., Levine, T. \& Behar, A. Malignant fubrous histiocytoma of the breast. Int Surg. 75, 63-66 (1990).

3. Tamir, G., Nobel, M., Hauben, D. J. \& Sandbank, J. Malignant fibrous histiocytoma of the breast. Eur J Surg Oncol. 21, 210-11 (1995).

4. Weiss, S. W. \& Enzinger, F. M. Malignant fibrous histiocytoma: an analysis of 200 cases. Cancer. 41, 2250-66 (1978).

5. Polinari, U., D’Ugo, D., Di Folco, S., Scambia, G. \& Sica, G. Malignant fibrous histiocytoma. Report of a case and review of the literature. Eur J Gynaecol Oncol. 6 105-08 (1985).

6. Pollard, S. G., Marks, P. V., Temple, L. N. \& Thompson, H. H. Breast sarcoma. A clinicopathologic review of 25 cases. Cancer. 66, 941-44 (1990).

7. Blanchard, D. K., Reynolds, C. A., Grant, C. S. \& Donohue, J. H. Primary nonphylloides breast sarcomas. Am J Surg. 186, 359-61 (2003).

8. Choudhury, M., Nangia, A., Singh, S. K., Pujani, M. \& Thomas, S. Cytohistomorphologic features of malignant fibrous histiocytoma of the breast: a case report. Acta Cytol. 54, 985-88 (2010).

9. Sheppard, D. G. \& Libshitz, H. I. Post-radiation sarcomas: a review of the clinical and imaging features in 63 cases. Clin Radiol. 56, 22-29 (2001).

10. Taghian, A. et al. Long-term risk of sarcoma following radiation treatment for breast cancer. Int J Radiat Oncol Biol Phys. 21, 361-67 (1991).

11. Cahan, W. G. et al. Sarcoma arising in irradiated bone; report of 11 cases. Cancer. 1, 3-29 (1948).

12. Biswas, S. \& Badiuddin, F. Radiation induced malignant histiocytoma of the contralateral breast following treatment of breast cancer: a case report and review of the literature. Cases J. 1, 313 (2008).

13. Gutman, H. et al. Sarcoma of the breast: implications for extent of therapy. The M. D. Anerson experience. Surgery. 116, 505-09 (1994).

14. van Niekerk, J. L., Wobbes, T., Holland, R. \& van Haelst, U. J. Malignant fibrous histiocytoma of the breast with axillary lymph node involvement. JSurg Oncol. 34, 32-35 (1987).
15. Jones, M. W., Norris, H. J., Wargotz, E. S. \& Weiss, S. W. Fibrosarcoma-malignant fibrous histiocytoma of the breast. A clinicopathological study of 32 cases. Am J Surg Pathol. 16, 667-74 (1992).

16. De Cesare, A. et al. Malignant fibrous histiocytoma of the breast. Report of two cases and review of the literature. Anticancer Res. 25, 505-08 (2005).

17. Gort, E. H. et al. Methylation of the TWIST1 promoter, TWIST1 mRNA levels, and immunohistochemical expression of TWIST1 in breast cancer. Cancer Epidemiol Biomarkers Prev. 17, 3325-30 (2008)

18. Nishimura, R. et al. Changes in the ER, PgR, HER2, p53 and Ki-67 biological markers between primary and recurrent breast cancer: discordance rates and prognosis. World J Surg Oncol. 9, 131 (2011).

19. Maciel Mdo, S. et al. $\mathrm{P} 53$ expression is a factor for prognostic assessment in breast sarcoma. Breast Cancer Res Treat. 71, 193-202 (2002).

20. Koo, J. S. \& Jung, W. Clinicopathlogic and immunohistochemical characteristics of triple negative invasive lobular carcinoma. Yonsei Med J. 52, 89-97 (2011).

21. Kafousi, M. et al. Immunohistochemical study of the angiogenetic network of VEGF, HIF1alpha, VEGFR-2 and endothelial nitric oxide synthase (eNOS) in human breast cancer. Pathol Oncol Res 18, 33-41 (2012).

\section{Acknowledgments}

This work was partly supported by special funds from Guangdong Provincial Key Research Laboratory of Breast Cancer Diagnosis and Treatment, grants from the National Key Basic Research Project of China (No.2011CB707705), the National Natural Science Foundation of China (No. 30973377) and the Natural Science Foundation of Guangdong Province (No. 10151503102000045).

\section{Author contributions}

S.Q.Q. collected patients' clinical records, performed the H\&E and immunohistochemical staining, and wrote the manuscript. X.L.W. and M.Y.W. contributed to the pathology studies. W.H.H. and Y.S.Q. collected patient information. Y.K.L. performed to imaging analysis. G.J.Z. designed the study and wrote the paper. S.Q.Q. and X.L.W. contributed equally to the work.

\section{Additional information}

Ethical approval: This study has been approved by the Ethics Committee of the Cancer Hospital of Shantou University Medical College, and was performed in accordance with the ethical standards laid down in the 1964 declaration of Helsinki and all subsequent revisions. All persons mentioned in the paper gave their informed consent prior to their inclusion in the study.

Competing financial interests: The authors declare no competing financial interests.

How to cite this article: Qiu, S.-Q. et al. Diagnostic and therapeutic strategy and the most efficient prognostic factors of breast malignant fibrous histiocytoma. Sci. Rep. 3, 2529; DOI:10.1038/srep02529 (2013).

(c) (1) (2) This work is licensed under a Creative Commons Attributioncc) visit http://creativecommons.org/licenses/by-nc-sa/3.0 